\title{
Blood stream infections with Candida species: a prospective study in a tertiary care center
}

\author{
Divakar A. ${ }^{1}$, Pillai M.G. ${ }^{2}$, A.K.V. ${ }^{3}$, Reddy R. ${ }^{4}$ \\ ${ }^{1}$ Dr. Arun Divakar, Assistant Professor, Department of General Medicine, ${ }^{2}$ Dr. M. Gopala Krishna Pillai, HOD \\ \& Professor, Department of General Medicine, ${ }^{3}$ Dr. Anil Kumar V, Assistant Professor, Department of \\ Microbiology, ${ }^{4}$ Dr. Rithesh Reddy Gundam, Post Graduate Student, General Medicine, Amrita Institute of \\ Medical Sciences, Ponekkara Kochi, Kerala, India. Affiliated to Amrita Vishwa Vidyapeetham, Kochi, Kerala, \\ India.
}

Corresponding Author: Dr. M. Gopala Krishna Pillai, HOD \& Professor, Amrita Institute of Medical Sciences, Ponekkara, Kochi, Kerala, India. Affiliated to Amrita Vishwa Vidyapeetham. E-mail Id: mgkpillai@aims.amrita.edu

\begin{abstract}
Aims and Objectives: To study the profile of blood stream infections with Candida species, their current epidemiological trend at our setting, clinical management, resistance trends and outcome in terms of mortality. Methods: This is a prospective observational study conducted among all adult in-patients, at AIMS, Kochi from August 1st 2014 to February 28th 2016, both in wards and ICUs, who have positive isolates of Candida species in bloodstream infection among the positive blood culture samples. Incidence rate of Candidemia was calculated and distribution of Candidemia with respect to characteristics like age, gender, comorbid illness, mechanical ventilation, prior antibiotic therapy, prolonged ICU stay. Results: Out of 1600 blood stream infection isolates, Candidemia was fourth most common blood stream isolate infection in our study with an incidence rate of $7.5 \%$. Candida tropicalis was the predominant species in our study (30.8\%). Mortality rate due to Candidemia was (61\%). Urethral catheterization, Central venous access, neutropenia at presentation, prior antibiotic therapy, renal failure, Dialysis patients, prolonged ICU stay were some of the risk factors found to be statistically significant in assessing the mortality of patients in our study with a $p$ value of $<0.001$. Conclusion: All patients with Candidemia should be treated with an antifungal agent at the earliest without any delay, as delay causes increase in mortality. All vascular catheters should be removed to help clear Candida from blood more quickly. Improved survival was found with the use of an Echinocandin and the removal of central venous catheters.
\end{abstract}

Keywords: Candida, Candidemia

\section{Introduction}

Candidemia describes the presence of Candidaspecies in the blood [1,2]. Invasive Candidiasis embodies a variety of diseases including Candidemia, disseminated Candidiasis, meningitis, and endo-phthalmitis. Most common form of invasive Candidiasis is Candidemia. Candidemia is a life-threatening affliction in most of the patients. Invasive Candidiasis has been of a concern as there is increased length and cost of hospitalizations as well as morbidities/ mortalities of patients $[1,3,4,5]$. Currently Candidemia is the

\footnotetext{
Manuscript rec eived: $10^{\text {th }}$ December 2017

Reviewed: $20^{\text {th }}$ December 2017

Author Corrected: $27^{\text {th }}$ December 2017

Accepted for Publication: $31^{\text {st }}$ December 2017
}

4th most common etiological agent nosocomial bloodstream infections [6,7]. Certain ICU populations, especially HIV and burn victims, are at even higher risk for Candida infection than is the typical ICU patient. Hence, Candida in a blood culture should never be viewed as a contaminant and should always prompt a search for the source of the bloodstream infection.

In the last 2 decades a change in the epidemiology of Candidemia has been noticed across the world $[8,9,10,11,12]$. With the change in the spectrum of Candida species causing Candidemia incidence of antifungal resistance of those yeasts are also on 
rise. With a high mortality rate of $35-75 \%$ early antifungal treatment is essential for survival $[13,14,15]$.

Objectives of Our Study- The primary objective: Incidence of bloodstream infections with Candida species among the total number of positive bloodstream infections in a tertiary care hospital in Kerala over a period of 1.5 year. Secondary objective is to study distribution of Candidemia with respect to characteristics like age, gender, comorbid illness, mechanical ventilation, prior antibiotic therapy, prolonged ICU stay, indwelling device, to study current pattern of antifungal susceptibility and to determine the outcome, in terms of ICU admission and mortality.

\section{Materials and Methods}

All adult in-patients admitted to Amrita Institute of Medical Sciences, Kochi, above 18 years of age, who have positive isolates of Candida in bloodstream infections among the positive blood culture samples received to the Microbiology department from August 1st 2014 to February 28th 2016, have been included in this study after getting written informed consent. A total of 1600 patient were included in this study.

Patients referred from another healthcare facility, having positive blood isolates of Candida and have been reconfirmed with Candidemia isolate on repeat blood sample test in Amrita Hospital will be included in the study population.

Patients in Medical ward as well as Medical ICU will be included in the study, provided they satisfy the other inclusion criteria.

\section{Original Research Article}

Inclusion criteria- Adult in-patients (age $>18$ years) from August 1 st 2014 to February $28^{\text {th }} 2016$, who have positive isolates of Candida in bloodstream. Patients referred from another healthcare facility, having positive blood isolates of Candida and have been reconfirmed with Candidemia isolate on repeat blood sample test. Patients in Medical ward as well as Medical ICU

Exclusion criteria- Patients with non-Candidemia yeast in blood isolate are excluded from the study.

Sample size- Based on the incidence rate of Blood stream infections with Candida among Adults, reported in an earlier publication(Bassetti M, Merelli M, Righi E et al) [3] and with 95\% of confidence and $20 \%$ allowable error, minimum sample size comes to 1600 samples of any infection among adults.

Ethics and Consent- Written informed consent was obtained from the study subjects participating in the study. There were no ethical or social dilemmas associated with this study and approval was taken from ethical committee before beginning the study.

Statistical Methods- Percentage incidence rate of incidence of blood stream infections with Candida among the total number of positive blood stream infections will be computed. To test the statistical significance of the association of various distributive factors of blood stream infections, chi square test will be applied. To obtain the association between outcome and selected variables, chi-square test and continuity correction method were applied. $\mathrm{P}$ value of $<0.05$ was considered significant.

\section{Results}

Table-1: Frequency of Candida Species.

\begin{tabular}{|c|c|c|}
\hline Species & Frequency & Percentage \\
\hline Candida Albicans & 27 & $22.5 \%$ \\
\hline Candida haemolunii & 21 & $17.5 \%$ \\
\hline Candida parapsilosis & 27 & $22.5 \%$ \\
\hline Candida tropicalis & 37 & $30.8 \%$ \\
\hline *Other Non Candida Albicans & 8 & $6.7 \%$ \\
\hline Total & 120 & $100 \%$ \\
\hline
\end{tabular}

*Other Non-Candida Albicans Include: C.Famata, C.Glabrata, C.Guilliermondii, C.Rugosa And C.Krusei Results of the study are recorded in the tables as listed here 
Table -1: Frequency of Candida Species

Table -2: Mortality Rate Due To Candidemia in Patients Who Were Treated With Different Antifungal

Table-3: Survival Rate in Candidemia Patients With Relation To ICU Stay

Table -4 Multivariate Analysis for Significance

Analysis of the observations are presented in the following figures

Figure -1 Survival Rate in Patients with Candidemia

Figure-2 Mortality Rate Due To Candidemia According To Species Distribution

Table-2: Showing Mortality Rate Due To Candidemia in Patients Who Were Treated With Different Antifungal.

\begin{tabular}{|c|c|c|c|c|c|}
\hline \multirow{3}{*}{ Treatment Given } & \multicolumn{4}{|c|}{ Outcome } & \multirow[t]{3}{*}{ p Value } \\
\hline & \multicolumn{2}{|c|}{ Death } & \multicolumn{2}{|c|}{ Alive } & \\
\hline & $\mathbf{n}$ & $\%$ & $\mathbf{n}$ & $\%$ & \\
\hline Amphotericin [14] & 5 & $35.7 \%$ & 9 & $64.3 \%$ & \multirow{5}{*}{0.115} \\
\hline Caspofungin [11] & 9 & $81.8 \%$ & 2 & $18.2 \%$ & \\
\hline Fluconazole [77] & 48 & $62.3 \%$ & 29 & $37.7 \%$ & \\
\hline Micafungin [7] & 3 & $42.9 \%$ & 4 & $57.1 \%$ & \\
\hline Voriconazole [11] & 8 & $72.7 \%$ & 3 & $27.3 \%$ & \\
\hline
\end{tabular}

Table-3: Showing Survival Rate in Candidemia Patients With Relation To ICU Stay.

\begin{tabular}{|c|c|c|c|c|c|}
\hline & $\begin{array}{c}\text { Duration of ICU } \\
\text { Stay }\end{array}$ & Succumbed & Survived & Total & $P$ Value \\
\hline \multirow{3}{*}{ ICU Stay } & $</=7$ Days & $22(39.3 \%)$ & $34(60.7 \%)$ & $56(100 \%)$ & \multirow{3}{*}{$<0.001$} \\
\hline & $>7$ Days & $51(79.7 \%)$ & $13(20.3 \%)$ & $64(100 \%)$ & \\
\hline & Total & $73(60.8 \%)$ & $47(39.2 \%)$ & $120(100 \%)$ & \\
\hline
\end{tabular}

Table-4: Multivariate Analysis.

\begin{tabular}{|c|c|c|c|c|}
\hline \multirow{2}{*}{ Duration of ICU stay } & \multirow{2}{*}{ Significance } & \multirow{2}{*}{ Odds Ratio } & \multicolumn{2}{|c|}{ 95\% C.I For O.R } \\
\cline { 3 - 5 } & & & Lower & Upper \\
\hline Urethral catheterisation & 0.001 & 22.078 & 3.804 & 128.125 \\
\hline Mechanical ventilator & 0.042 & 4.305 & 1.056 & 17.544 \\
\hline ICU Stay & 0.000 & 15.509 & 3.817 & 63.006 \\
\hline
\end{tabular}

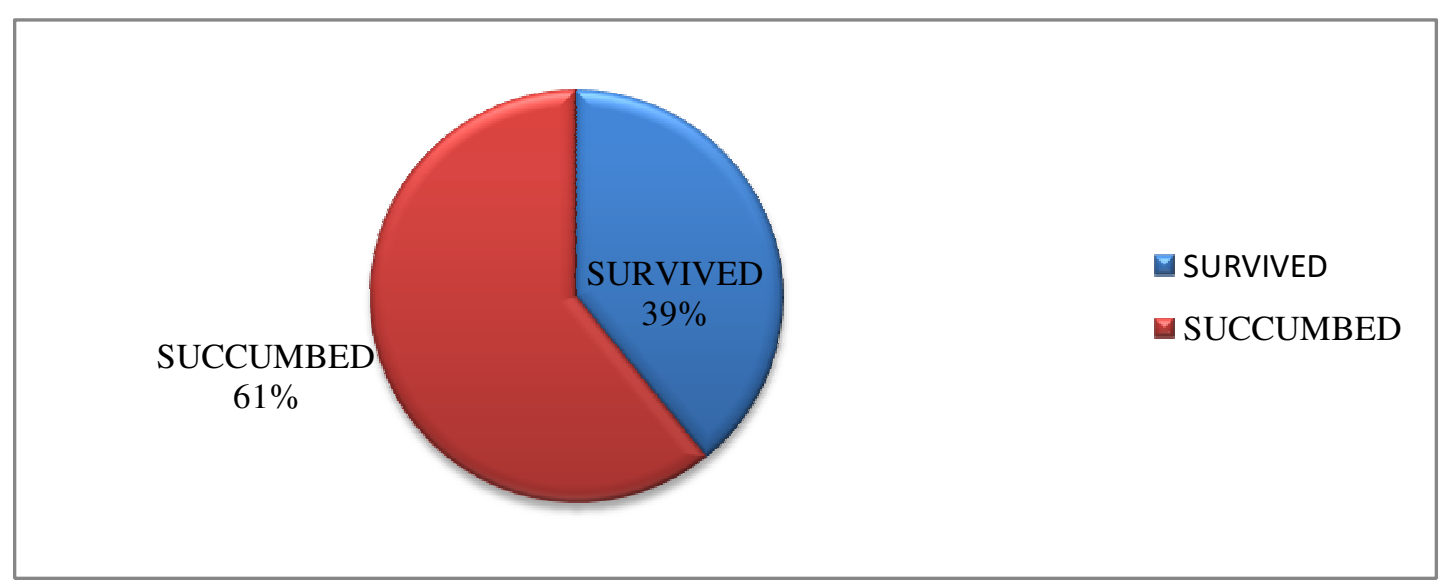

Figure-1: Survival Rate in Patients with Candidemia 
Mortality rate due to candidemia according to species wise distribution*Other Non Candida Albicans include: C.famata, C.glabrata, C.guilliermondii, C.rugosa and C.Krusei

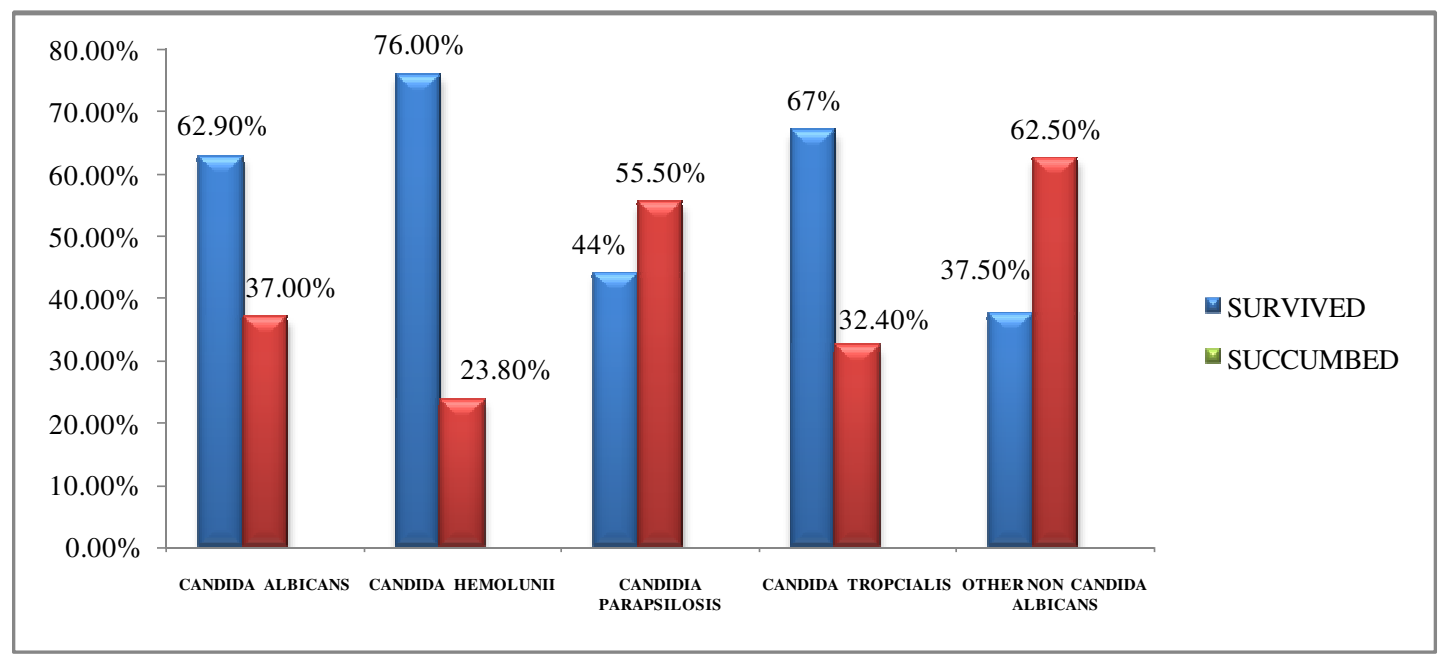

Figure-2: Mortality Rate Due To Candidemia According To Species Distribution

\section{Discussion}

We found an overall incidence of 120 cases of Candidemia among 1600 positive blood culturesover 1.5 year with an incidence of $7.5 \%$. Candidemia was fourth most common blood stream isolate infection in our study after Klebsiella pneumonia, Escherichia coli and Staphylococcus aureus. In an another prospective, nationwide, multicenter, observational study was conducted at 27 Indian ICUs from April 2011 to September 2012 in India, 1400 Candidemia cases were isolated. A study done in SGPGI Lucknow in 2002 found to have $1.61 \%$ incidence of blood stream infections due to Candidemia and it was $8^{\text {th }}$ most common blood stream infection. In Gangaram hospital, the incidence was $1.74 \%$, while in Rohatk medical college it was $8.1 \%, 6 \%$ in AIIMS, and $6.9 \%$ in MAMC New Delhi. Most of the people with Candidemia in our study were $>60$ years old $(39.2 \%)$, which was in concordance with other studies where most of the Candidemia isolates belonged to patients with mean age group between $59.0-66.2$ years $[16,17]$.

Most of the patients with Candidemia in our study were Males (60.8\%).Patients with Candida non albicans (93) were more compared to Candida albicans (27) in our study.Candida tropicalis $(30.8 \%)$ was the predominant species in our study.This is in contrast to the developed world, where $C$. tropicalisis uniformly less common (5.6$12.0 \%$ ) [3-7], and C. albicans (45.0-74.0\%) and $C$. glabrata (16.7-22.6\%) are more prevalent
$[18,19,20]$ where as we have encountered $C$. glabrata Candidemia in only $0.83 \%$ of patients. In an earlier study at the coordinating centre, $82 \%$ of health care providers were found to carry yeast on their hands and $80 \%$ were C. tropicalis[11]. Therefore, horizontal transmission and compromise of infection control systems are distinct possibilities for the high rate of C.tropicalis candidemia in India. The high rate of C.tropicalis candidemia was observed in other Asian countries as well [21]. Proper hygienic measures are required to curb the incidence of horizontal spread of Candidemia. Most of the Cases of Candidemia were admitted under General Medicine department $(30 \%)$. Co-morbidities of our patients are similar to a certain extent to earlier reports. In the current study frequency of Candidemia was higher in Diabetic population $(63.3 \%)$. Earlier studies have showed that they have also reported sizable proportions of diabetes $(10.7-28.0 \%)$ in their Candidemia population $[1-3,6,9,10,13,14] .5 \%$ of our patients with Candidemia had an underlying hematological malignancy, which was less compared to other studies where the incidence was $24.6-36.1 \%[2,14]$.

Patients with Candidemia who had HIV infection in our study were only $1.7 \%$, which was almost in concordance with other studies, where the frequency was $4.0-6.0 \%$. [22,23,24,25]. About $9.2 \%$ of the patients with Candidemia in our study were neutropenic at presentation, which was almost 
Original Research Article

similar to other studies where the frequency was $6.6-19.7 \%$ [4, 6, 26,27,28,29]. Only $1.7 \%$ of the patients in our study with Candidemia had a prior abdominal surgery, which was less compared to $44.7-66.1 \%$ seen in other series of studies[1,2]. Among types of surgery, abdominal surgery is considered a leading risk factor for Candidemia [15]. Central venous catheterization was present in $66.7 \%$ of the patients with Candidemia in our study which was almost in concordance with other studies where the incidence was found to be 88.5$100 \%$ in other studies $[1,14]$. Invasive mechanical ventilator was present in $49.2 \%$ of the patients with Candidemia in our study as compared to 72.1$97.4 \%$ in other studies $[1,14]$. Urinary catheterization was present in $73.3 \%$ of the patients with Candidemia in our study compared to 86.7-97.4 \% in other studies [1,10], which was almost in concordance with our study.

Frequency of Candidemia in patients who were on Hemodialysis was $19.22 \%$ compared to $17.5-$ $32.5 \%[3,9]$ in other studies, which was in concordance with our study. Frequency of Candidemia in patients who were on TPN was $15 \%$ which was less compared to $43.7-71.1 \%$ in other studies [1,3]. Candidemia was present in $36.7 \%$ of patients in our study who received prior antibiotic therapy, $31.7 \%$ of patients who had prior renal failure, $6.7 \%$ of patients who received prior corticosteroid therapy, $1.7 \%$ of patients who received solid organ transplant therapy. 73 people among 120 people in our study (60.8\%) with Candidemia succumbed to their illness which is higher compared to the EPIC II international series where they found a mortality of $42.6 \%$ [14]. Mortality rates vary across the globe, they vary between $35-75 \%$ [1]. Candidemia-attributable mortality also varies widely $(5.0-49.0 \%)$ between centers $[1,2,5,16]$.

Urethral catheterization, Central venousaccess, Mechanically ventilation, Neutropenia at presentation, prior antibiotic therapy, renal failure, Dialysis patients, on TPN, prolonged ICU stay were some of the risk factors which were found to be statistically significant in our study while assessing the mortality of patients in our study with a $p$ value of $<0.001$. In our study 3 variables were found to be significant while assessing the outcome in terms of mortality, they are Urethral catheterization, Mechanical ventilator and ICU stay. If patients were urethrally catheterized, there was 22 times more chance of succumbing to their illness due to Candidemia than if they were not urethrally catheterized. If patients were on mechanical ventilator, there was 4 times more chance of succumbing to their illness due to Candidemia than if they were not mechanically ventilated. If patients had prolonged ICU stay, then there were 15 times more chance of succumbing to their illness due to Candidemia than if they did not have any ICU stay.

\section{Conclusion}

We conclude with an inference that all patients with Candidemia should be treated with an antifungal agent at the earliest, including patients who have only one blood culture that yields Candida and those with a vascular catheter tip that yields Candida. Any delay in initiation of treatment, showed an increase in mortality rates. All vascular catheters should be removed because removal has been shown to help clear Candida from blood more quickly. Repeated blood cultures should be obtained to ascertain that the fungemia has resolved, and treatment should continue for 2 weeks after the date of the first negative blood culture. Improved survival and clinical success was found with the use of an echinocandin and the removal of central venous catheters.

\section{Funding: Nil, Conflict of interest: None Permission of IRB: Yes}

\section{References}

1. Gonzalez de Molina FJ, Leon C, Ruiz- Santana S et al (2012) Assessment of candidemia-attributable mortality in critically ill patients using propensity score matching analysis. Crit Care 16(3):R105 PMID:22698004 PMCID: PMC3580662DOI:10. $1186 / \mathrm{cc} 11388$.

2. Bassetti M, Merelli M, Righi E et al. Epidemiology, species distribution, antifungal susceptibility, and outcome of candidemia across five sites in Italy and Spain. J ClinMicrobiol. 2013; 51 (12):4167-4172DOI:10.1128/JCM.01998-13.

3. Playford EG, Marriott D, Nguyen Q et al. Candidemia in non-neutropenic critically ill patients: risk factors for non-albicans Candida spp. Crit Care Med.2008; 36(7): 2034-2039 PMID: 18552700DOI:10.1097/CCM. 0b013e 3181760f42. 


\section{Original Research Article}

4. Pappas PG, Rex JH, Lee $\mathrm{J}$ et al. A prospective observational study of candidemia: epidemiology, therapy, and influences on mortality in hospitalized adult and pediatric patients. Clin Infect Dis 2003; 37(5):634-643PMID:12942393doi:10.1086/376906

5. Chen S, Slavin M, Nguyen Q et al. Active surveillance for candidemia,Australia.EmergInfectDis20 06;12(10):1508-1516doi:10.3201/eid1210.060389

6. Leroy O, Mira JP, Montravers P et al (2010) Comparison of albicans vs. non-albicans candidemia in French intensive care units. Crit Care 14 (3): R98https://doi.org/10.1186/cc9033

7. Pfaller MA, Messer SA, Moet GJ et al (2011) Candida bloodstream infections: comparison of species distribution and resistance to echinocandin and azole antifungal agents in intensive care unit (ICU) and non-ICU settings in the SENTRY antimicrobial surveillance program (2008-2009). Int J Antimicrobial Agents 38(1):65-69 PMID: 21514797 DOI:10.1016/j. ijantimicag.2011. 02.016

8. Meyer E, Geffers C, Gastmeier P et al (2013) No increase in primary nosocomial candidemia in 682 German intensive care units during 2006-2011. Euro Surveill 18(24):20505PMID:23787164.

9. Chow JK, Golan Y, Ruthazer R et al (2008) Risk factors for albicans and non-albicans candidemia in the intensive care unit. Crit Care Med36 (7):19931998 PMID:18552702 DOI:10.1097/CCM.0b013e $31816 \mathrm{fc} 4 \mathrm{~cd}$.

10. Leroy $\mathrm{O}$, Gangneux JP, Montravers $\mathrm{P}$ et al (2009) Epidemiology, management, and risk factors for death of invasive Candida infections in critical care: a multicenter, prospective, observational study in France (2005-2006). Crit Care Med 37(5):1612-1618 1618 PMID:19325476 DOI:10. 1097/ CCM.0b013e31819efac0.

11. Chakrabarti A, Chatterjee SS, Rao KL et al (2009) Recent experience with fungemia: change in species distribution and azole resistance. Scand J Infect Dis 41(4):275-284.PMID:19229762 DOI:10. 1080/ 00365540902777105.

12. Mootsikapun P, Hsueh PR, Talwar D et al (2013) Intravenous anidulafungin followed optionally by oral voriconazole for the treatment of candidemia in Asian patients: results from an openlabel phase III trial. BMC Infect Dis 13:219https: // doi.org/10.1186/1471-2334-13-219.

13. Blot SI, Vandewoude KH, Hoste EA et al (2002) Effects of Nosocomial Candidemia on outcomes of critically ill patients. Am J Med 113 (6): 480-485. PMID:12427497.

14. Kett DH, Azoulay E, Echeverria PM et al (2011) Candida bloodstream infections in intensive care units: analysis of the extended prevalence of infection in intensive care unit study. Crit Care Med 39 (4): 665-670. PMID:21169817 DOI: 10. 1097 / CCM.0b013e318206c1ca.

15. Leon C, Ostrosky-Zeichner L, Schuster M (2014) What's new in the clinical and diagnostic manage- ment of Invasive Candidiasis in critically ill patients. PMID:24718642 DOI:10.1007/s00134014-3281-0.

16. Gudlaugsson O, Gillespie S, Lee K et al (2003) Attributable mortality of nosocomial candidemia, revisited. Clin Infect Dis 37(9):1172-1177. PMID: 14557960 DOI: $10.1086 / 378745$.

17. Fraser VJ, Jones $M$, Dunkel $J$, et al. Candidemia in a tertiary care hospital: epidemiology, risk factors, and predictors of mortality. Clin Infect Dis 1992; 15:414. PMID: 1520786.

18. Quindos G. Epidemiology of candidaemia and invasive candidiasis. A changing face. Rev Iberoam Micol. 2014;31:42-48. PMID:24270071 DOI: 10. 1016/j.riam.2013.10.001.

19. Bitar D, Lortholary O, Le Strat Y, Nicolau J, Coignard B, Tattevin $\mathrm{P}$, et al. Population based analysis of invasive fungal infections, France, 2001-2010. Emerg Infect Dis 2014;20:1149-55. PMID: 24960557PMCID: PMC4073874 DOI:10. 3201/eid2007.140087

20. Hidron AI, Edwards JR, Patel J, Horan T, Sievert DM, Pollock DA, et al. Antimicrobialresistant pathogens associated with healthcareassociated infections: annual summary of data reported to the National Healthcare Safety Network at theCentersforDiseaseControlandPrevention,2006 e2007.Infect Control Hospl Epidemiol 2008; 29: 996e1011.PMID:18947320DOI: 10. 1086/ 591861. 
21. Fridkin SK. The changing face of fungal infections in health care settings. Clin Infect Dis 2005; 41:1455.https://doi.org/10.1086/497138.

22. Wisplinghoff $\mathrm{H}$, Bischoff $\mathrm{T}$, Tallent SM, Seifert Wenzel RP, Edmond MB. Nosocomial blood stream infections in US hospital: analysis of 24,179 cases from a prospective nationwide surveillance study. Clin Infect Dis 2004; 39:309-17. https:// doi.org/10.1086/425328

23. Marchetti O, Bille J, Fluckinger U, Eggiman P, Ruef C, Garbino J, et al. Epidemiology of candidemia in Swiss tertiary care hospitals: secular trends, 1991-2000. Clin Infect Dis 2004; 38:31120. https://doi.org/10.1086/380637

24. Gudlaugsson O, Gillespie S, Lee K, Vande Berg J, Hu J, Messer S, et al. Attributable mortality of nosocomial candidemia, revisited. Clin Infect Dis 2003; 37:1172-7. PMID: 14557960 DOI:10. $1086 / 378745$.

25. Morgan J, Meltzer MI, Plikatys BD, Sofair AN, Huie-White S, Wilcox S, et al. Excess mortality, hospital stay and cost due to candidemia: a casecontrol study using data from population-based
Original Research Article

candidemia surveillance. Infect Control Hosp Epidemiol 2005; 26:540-7. PMID: 16018429DOI: 10. $1086 / 502581$

26. Liu CY, Liao $\mathrm{CH}$, Chen YC, Chang SC. Changing epidemiology of nosocomial bloodstream infections in 11 teaching hospitals in Taiwan between 1993 and 2006. J Microbiol, Immunol Infect 2010;43:416e29. Journal of Microbiology, Immunology and Infection (2015) 48, 306315

27. Hobson RP. The global epidemiology of invasive Candida infections. Is the tide turning? J Hosp Infect 2003;55:159e68. PMID:14572481.

28. Ruan SY, Hsueh PR. Invasive Candidiasis overview: An overview from Taiwan. J Formos Med Assoc 2009; 108:443e51. PMID:19515624 DOI:10. 1016/S0929-6646(09)60091-7.

29. Horn DL, Neofytos D, Anaissie EJ, Fishman JA, Steinbach WJ, Olyaei AJ, et al. Epidemiology and outcomes of candidemia in 2019 patients: data from the prospective antifungal therapy alliance registry. Clin Infect Dis 2009;48:1695e703. PMID: 19441981DOI:10.1086/599039.

\section{How to cite this article?}

Divakar A, Pillai M.G, A.K.V, Reddy R. Blood stream infections with Candida species: a prospective study in a tertiary care center. Int J Med Res Rev 2017;5 (12):1015-1021.doi:10.17511/ijmrr. 2017.i12.07 